\title{
Handling Toll Traffic Using Rfid In Highway Scenario Environment
}

\author{
Rupendra kumar $^{1}$, Awadhesh Kumar ${ }^{2}$ \\ M.Tech Scholar, CSE, Kamla Nehru Institute of Technology, Sultanpur, U.P. \\ ${ }^{2}$ Associate Professor, CSE, Kamla Nehru Institute of Technology, Sultanpur, U.P.
}

\begin{abstract}
In this research paper, we consider the wireless sensor networks (WSNs) in toll road using RFID. Sensor network are collection of sensor node and they co-operatively send sensed data to base station. In a real time scenario all the nodes follow the traditional toll tax rule and payment done manually. Therefore, we try to create a system for radio frequency identification (RFID) technology for electronic toll collections (ETC) system for online payment and provide a smooth run through. Current RFID tags appear in a public environment like vehicle systems (toll collection or car key) applications and retail supply chains. The RFID tags are enhanced with additional functionality for sensing and localization. In this paper the evolution of wireless sensor networks (WSNs) will be presented, using RFID techniques with examples of implementation in toll traffic using RFID in the highway scenario environment.
\end{abstract}

Keywords: RFID, Tags, Antennae, WSNs

\section{Introduction}

Wireless sensor networks are a wireless network, which consists of a base station and a number of a wireless sensor node. In this wireless sensor node, its sense data and send to base station. As sensor nodes are battery driven, these are usually capable of measuring the physical phenomenon like temperature, sound, vibration, pressure, etc. Sensor nodes are deployed for gathering data; this work drains more battery power which is more expensive. In this sensor networks present an increasingly Sensor node need less power for processing as compared to transmitting data. Another quality of sensor networks is the group of sensor nodes to produce high-quality information about the sensing environment. The features of sensor networks provide a large collection of applications such as health, military, and home. While various protocols and algorithms have been proposed for consistent wireless ad-hoc networks and also not suitable to the special features and application requirements of sensor networks. Then, many routing and data distribution protocols should be designed for sensor networks where the following issues should be measured: Energy Awareness, MAC for Wireless Sensor Networks, Time Synchronization, Power-saving Mode of Operation, and Routing.

Here sensor nodes perform both as a router as well as a normal sensing node. Base station collects the information from all sensor nodes, evaluate it and perform some computation which yields information that are human understandable. At this WSNs base station have unlimited source of energy powering so it should implement different algorithms and protocols which result in improvement in lifetime of sensor node and network lifetime as well as energy efficient.

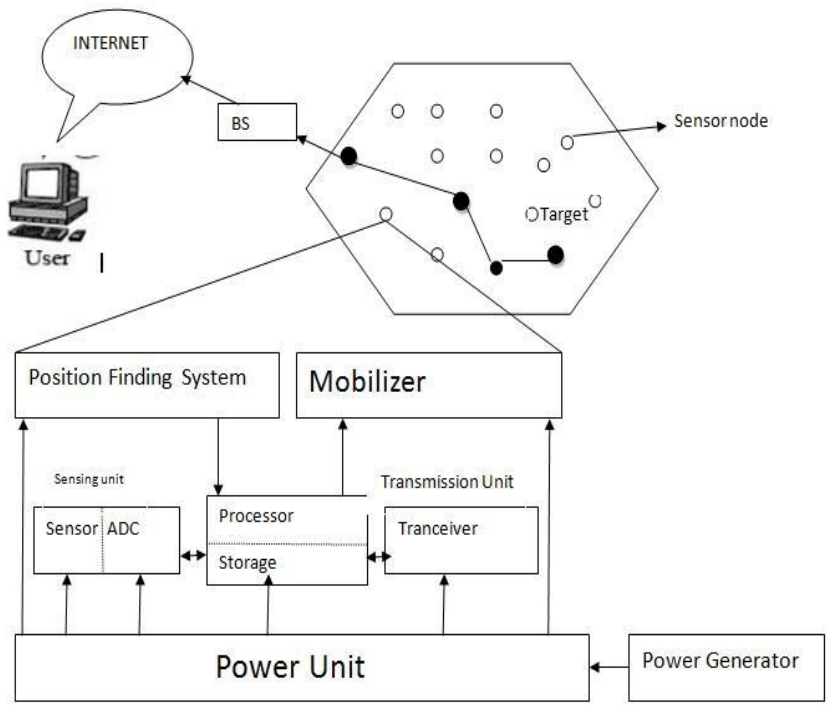

Fig.1: An Architecture of WSNs. 
In this paper consider a toll booth. Millions of drivers/consumer pass through toll booths paying toll tax. The past of time toll payment system was manual and consumers are using a manual system using coins or cash by hand to cross the toll plaza gate. In a manual process means too much time consuming and consumers have to wait in a row for a long time for crossing the toll plaza. In waiting time and fuel of the vehicle is also consumed. In a present time this manual system changes to an automated system. Where consumer no wait for pay cash or get token to cross the toll plaza. This automatic system used the technology of RFID. In this term of RFID means Radio frequency identification (RFID) technology is a non-contact method of item identification based on the use of radio waves to communicate data concerning an item between a tag and a reader.

Radio Frequency Identification (RFID) is an auto identification technology. Which uses Radio Frequencies (between $30 \mathrm{kHz}$ and $2.5 \mathrm{GHz}$ ) to identify objects remotely. In this system does the job of notice, billing and accounting for vehicles as they pass through a toll gate using RFID as the identification technology. The tag of RFID is programmed with information in the form of an Electronic Product Code (EPC). Which can be read over a significant distance so that its contents identify the vehicle and improve an operation to be undertaken with respect to the particular tag identity taking advantage of radio frequencies. In this system provide a facility to travel longer ranges with better data capacities and high speed attained with maximum accuracy. RFID is one of important system solutions for tracking and tracing objects both globally and locally using RFID tags. In this auto-ID procedure for identifying objects automatically within a geographical area. The uses of RFID tags which communicate information by radio wave through antennae on small computer chips attached to objects so that such objects may be identified, located, and tracked.

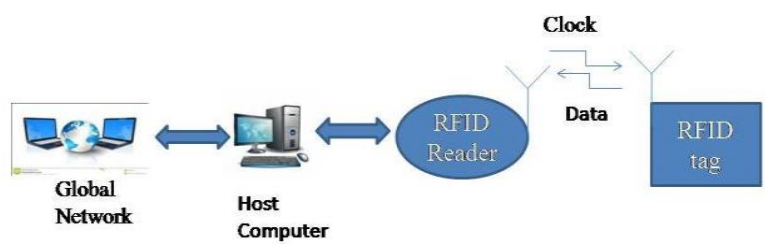

Fig.2: An Architecture RFID

In RFID systems, the tags that hold the data are broken down into two different types Passive and Active. Passive tags do not have batteries and have indefinite life expectancies. Second one is Active Tags means powered by batteries and either have to be recharged, have their batteries replaced or be disposed of when the batteries fail. In an RFID tag used two types of chips: Read-Only and Read-Write. Read-only chips are considered to programmed with unique information stored on them that cannot be changed. Read-Write chips means the user can add information to the tag or write over presents information when the tag is within range of the reader.

\section{Related Work}

The related work regarding our Proposed System is introduction of RFID technology in the nodes. RFID technology is an identification technology in which every node is equipped with RFID tags to remotely identify by RFID. RFID technology uses Radio Frequencies for communication and identification of RFID tag and uses radio frequencies (between $30 \mathrm{kHz}$ and $2.5 \mathrm{GHz}$ ) to identify objects remotely. RFID technology used to monitor and controlling all the nodes that approaches to the toll tax. Active and passive tags are used in the RFID technology. This technology is a non-contact method of item identification based on the use of radio waves to communicate data about an item between a tag and a reader. The range of the reader is dependent upon its operational frequency.

In existing system that is no concept of RFID readers, so all the node to the toll tax and do the normal procedure of payment. As the node approaches the identification site, the computerized control unit placed near toll lane receives the identifier signal and calculates the toll to be debited and electronically debits the toll on the account of the particular nodes. This result in error congestion and fuel of nodes is also consumed. Since we cannot differentiate between RFID nodes and non RFID nodes, there is no benefit for RFID nodes. Modern nodes are equipped with the latest technology, devices and other things, but due to less supportable infrastructure they unable to use this technology. This is one of the major drawbacks with latest equipped nodes.

\section{Proposed System}

In our propose system we are trying to utilize the embed technology of node and give benefits to these nodes. As we know latest nodes equipped with an RFID tag. RFID tags are mainly of two types i.e., active RFID tag and passive RFID tag. Active RFID tags are RFID tag which requires a power source either through power infrastructure or battery stored mechanism. In this type of tags the number of read operations performed by RFID tags is limited by the power supply. So its operation is limited on active tags while Passive RFID tags 
have an indefinite operational life and perfect to embed in the node. It mainly consists of three units antenna, semiconductor chip attached to an antenna and encapsulation unit. The tag readers activates the communication with RFID tag while the antenna captures the energy related to tag id. Encapsulation unit is used for maintaining integrity of the system. In our proposed system we design two sink nodes S1 and S2. Sink node (S1) is placed before the $800 \mathrm{~m}$ of toll plaza while second Sink node (S2) is located at toll plaza. The First Sink node (S1) is used for checking and diverting of the RFID tag node from non-RFID tag node. In our proposed we create two lanes, i.e. RFID node lane and simple lane for the non -RFID node. In RFID-lane the RFID tag node pays their toll taxes online while in normal lane a node pay their toll taxes by halting at toll tax and pay their toll fares and then depart.

The working of our proposed system is such that these RFID tags put on the windshield of nodes. So when these nodes come near RFID readers of Sink Node (S1) terminal, then RFID readers read the RFID tag of node to distinguish whether it is an RFID tag node or NON-RFID tag node. Once it checks and find types of nodes, it signals node to follow defined lane for toll tax payment. If it finds an RFID tag node, it sends a message to node to follow RFID lane otherwise it send messages to follow the normal lane. When an RFID tag node follows RFID lane, before reaching Sink node (S2), the RFID tag nodes can make payment online if they haven't already paid toll tax. Sink node (S2) is used for monitoring and tracking of RFID tag nodes for payment queries. Sink node (S2) also maintained database for each RFID tag node for further payment related queries. As soon as RFID tag node approaches Sink nodes (S2), sink nodes (S2) checks whether nodes have made its payment or not through its database. If the payment is already done simply pass that node without halting or message generation. If not it generates a message to node for payment. If a node does not make its payment before reaching a toll tax barrier, it stops the nodes and allow the node to pay at that same time before the lifting toll barrier.

Our proposed System can be described with our proposed algorithm which as follows:-

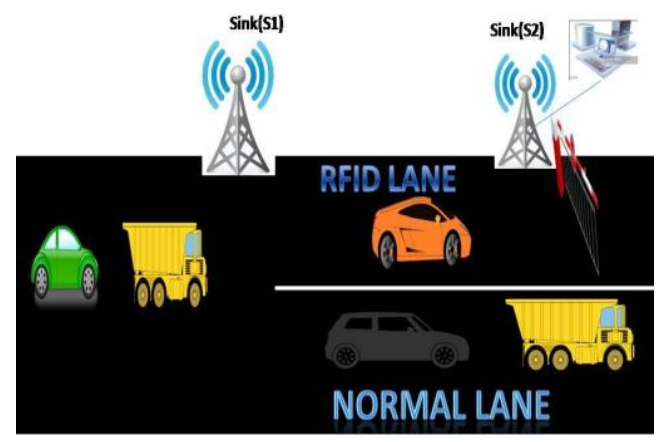

Fig.3: Propose System Scenario

\section{Flow Chart}

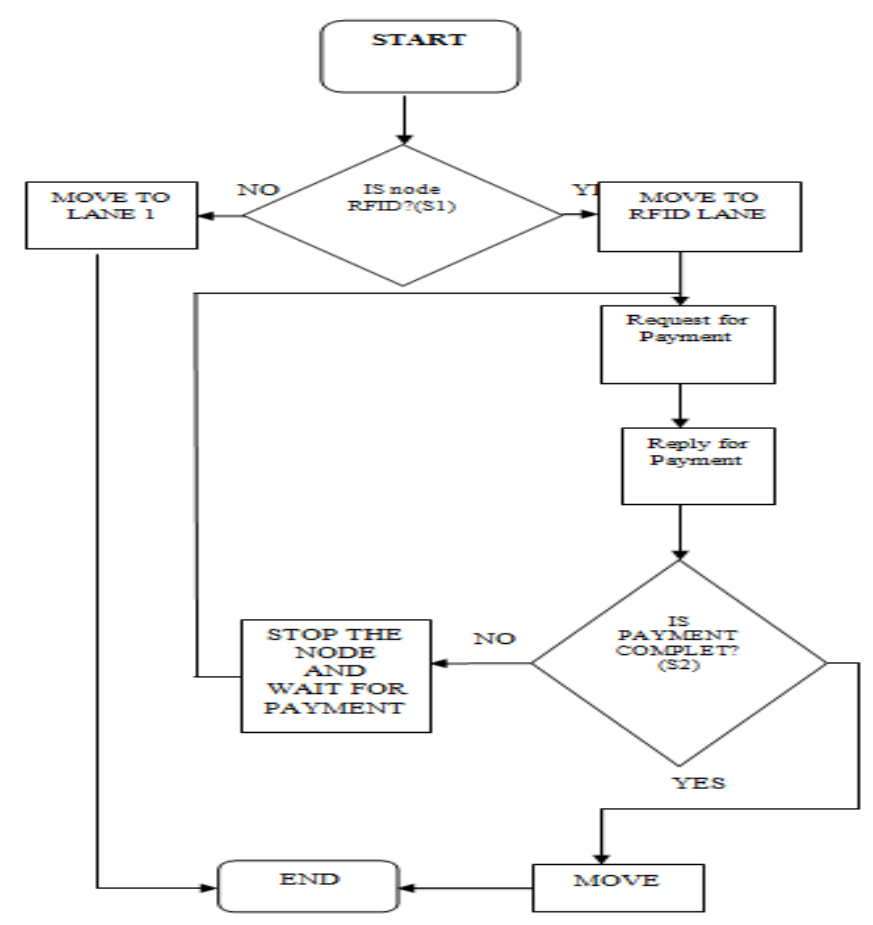


The above flow chart can be explained with the following steps:-

Step1:Assign RFID node $=1$ and Non RFID node $=0$.

Step2: Sense whether the node approaching to sink node (S1) is RFID node or Non-RFID node based on their value.

Step3: If it is an RFID node move to RFID lane.

a) Check Payment Status of RFID node at the sink node (S2) if yes; allow that node to pass toll tax.

b) If not Stop that node and wait until payment is made manually.

Step 4: Else move to Lane 1.

Step5: Repeat step 2 to 4 until all nodes are not sensed and passed through toll plaza.

\section{Simulation Result And Analysis}

We simulate our propose system in NS2 (Network Simulator 2). In this simulation, we consider N*M sq.m simulation area and packet size 50bits/sec. In a our propose system we apply AODV and DSDV routing protocol and compare results in terms of throughput, end to end delay and packet delivery ratio. We perform in NS2 simulation for 10 nodes, 20 nodes, 30 nodes, 40 nodes and 50 nodes and compute performance metrics for all these numbers of nodes. In NS2 simulation, we perform a computation process by using trace file and AWK programs. We consider following parameter for Simulation Configuration.

Table 1: Simulation Parameters.

\begin{tabular}{|l|l|}
\hline Parameter & Value \\
\hline Routing Protocol & AODV, DSDV \\
\hline Simulation time & T time $(\mathrm{ms})$ \\
\hline Number of Nodes & N nodes \\
\hline Channel type & Wireless sensor \\
\hline Topography Dimension & $\mathrm{N}^{*} \mathrm{M}$ sq.m \\
\hline Queue Length & 50 \\
\hline Speed & $50 \mathrm{~m} / \mathrm{s}$ \\
\hline MAC protocol & IEEE 802.11 \\
\hline
\end{tabular}

\section{Reduced Time At Toll Barrier}

Let there are $\mathrm{n}$ nodes approaching to toll plaza barrier in our existing system. If at the toll barrier if a node takes t1 units of time to the cross toll barrier, Then total time taken by all nodes to cover toll plaza barrier $=(\mathrm{n} * \mathrm{t} 1)$ units.

Now consider our Proposed System, here there are two lanes, one for RFID lanes and other is NonRFID lane. Since in our proposed system we directly pass RFID node on RFID lanes without halting. While Non-RFID lane will take simply same time as our existing system, i.e. $t 1$ units. If the total $\mathrm{n}$ nodes approach to toll barrier and we assume $\mathrm{n} 1$ ( $\mathrm{n}>\mathrm{n} 1)$, are RFID nodes

Then Total time taken by all nodes to cross toll barrier $=(n 1 * t 2)+\{(n-n 1) * t 1\}$

Since $\mathrm{t} 2=0$

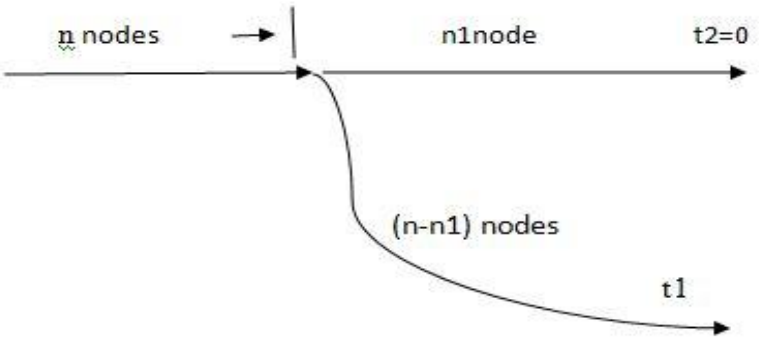

Fig.4 Scenario of proposed system

Total time taken by all nodes to cross toll barrier $=(n 1 * t 2)+\{(n-n 1) * t 1\}$

$=\{(\mathrm{n}-\mathrm{n} 1) * \mathrm{t} 1\}$

So our proposed system results less time taken by all nodes to cross the toll barrier than our existing system.

For example, we assume $40 \%$ of total nodes are RFID nodes and each nodes halt at the toll plaza for $\mathrm{t} 1=2$ units. Since, in the existing system, there is no different lane for RFID node so all RFID nodes follow the same procedure of toll payment. .While in the proposed system, there separates RFID lane for RFID node. 
Table 2: Time Comparison between existing system and proposed system

\begin{tabular}{|l|l|l|l|}
\hline $\begin{array}{l}\text { Total Number } \\
\text { of Nodes }(\mathrm{n})\end{array}$ & $\begin{array}{l}\text { RFID nodes } \\
(40 \% \text { of Total Nodes) }\end{array}$ & $\begin{array}{l}\text { Time taken by total nodes to } \\
\text { cross the toll plaza (Existing) }\end{array}$ & $\begin{array}{l}\text { Time taken by total nodes to } \\
\text { cross the toll plaza (Proposed) }\end{array}$ \\
\hline 10 & 4 & 20 & 12 \\
\hline 20 & 8 & 40 & 24 \\
\hline 30 & 12 & 60 & 36 \\
\hline 40 & 16 & 80 & 48 \\
\hline 50 & 20 & 100 & 60 \\
\hline
\end{tabular}

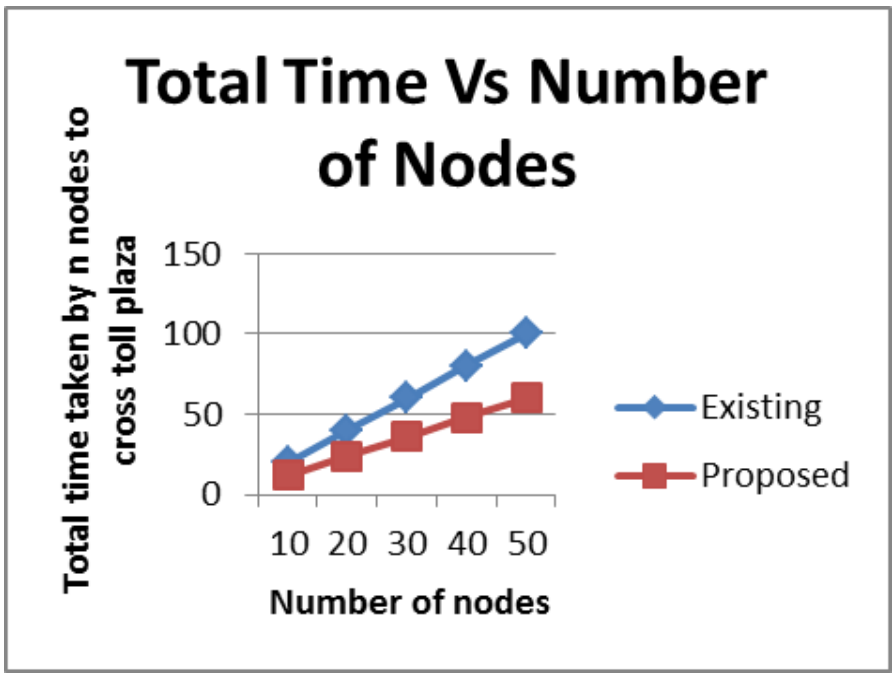

Fig.5: graph- proposed system and existing system

\section{PDR (Packet Delivery Ratio)}

In this paper the system ratio of number of packets received to number of packets sent. As figure we can say that PDR of AODV is much greater than DSDV.

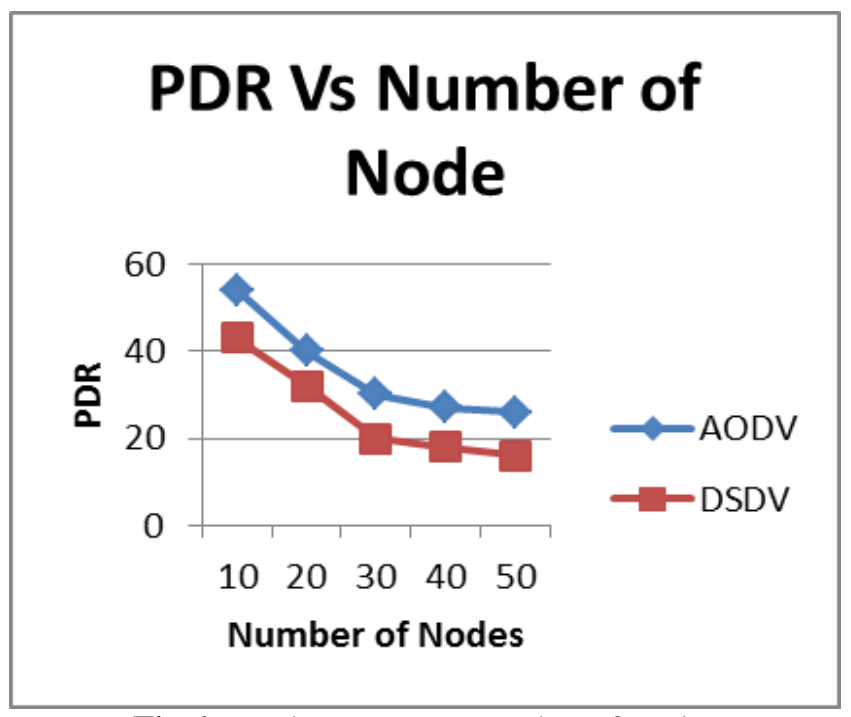

Fig.6: graph - PDR Vs. Number of Nodes

\section{Conclusion}

In our research paper development of RFID based toll traffic in highway scenario is provided that RFID technology has good results in implementing in different applications. In this research paper, we propose a system based on wireless sensor networks (WSNs) architecture. The Sink Node (S1) is used for detecting RFID tag node before the $800 \mathrm{~m}$ of toll plaza either it is active tag or passive tag and follow RFID-lane and non RFID lane. The function of sink Node (S2) checks the payment of RFID -lane node is paid or not in toll plaza database if it is paid then pass the node in the toll barrier without stopping. The benefits of this automation toll deduction system are over money loss in toll plaza by reducing manpower required for collection of money, reduce the traffic indirectly, and also safe fuels resulting in reduction of time at toll center. 


\section{Acknowledgment}

The authors wish to thank the faculty from the Computer Science and Engineering department at KNIT, Sultanpur for their continued support and feedback.

\section{References}

[1]. CHALUVADI.VENKATESWARLU, A.RAGHU RAM "Safer Cards Enhancing RFID Security and Privacy via Location Sensing" International Journal of Research In Advanced Engineering Technologies, vol.3, pp.31-37, 2014.

[2]. Mr. R. M. Hushangabade, Prof. S.V. Dhopte "Implementation Of Rats For The Purpose Of Vehicle Tracking \& Toll Tax Collection” International Journal Of Computer Science And Applications, Vol. 6, pp.92-96 , 2013.

[3]. Asif Ali Laghari, M. Sulleman Memon and Agha Sheraz Pathan "RFID Based Toll Deduction System" Information Technology and Computer Science, Vol 4, pp.40-46, 2012.

[4]. S.Nandhini, P.Premkumar, "Automatic Toll Gate System Using Advanced RFID And GSM Technology" International Journal of Advanced Research in Electrical,Electronics and Instrumentation Engineering, Vol.3, pp.13002-13006, 2014.

[5]. Divya Sharma , Kamal Kant “Design Analysis of RFID Automotive Traffic Security System Using ARM Platform”, International Journal of VLSI and Embedded Systems-IJVES, Vol.04 pp.357-363, 2013.

[6]. Kumar Chaturvedula .U.P, "RFID Based Embedded System for Vehicle Tracking and Prevention of Road Accidents", International Journal of Engineering Research \& Technology (IJERT), Vol. 1 Issue 6, ISSN: 2278-0181, 2012.

[7]. Venugopal Prasanth, Hari Prasad R., K.P. Soman, "Ticketing Solutions For Indian Railways Using RFID Technology", International Conference on Advances in Computing, Control, and Telecommunication Technologies, act, pp.217-219, 2009.

[8]. Rushi Vyas, Napol Chaisilwattana, "Paper-Based RFID-Enabled Wireless Platforms For Sensing Applications", IEEE TRANSACTIONS ON MICROWAVE THEORY AND TECHNIQUES, Vol.57, No. 05, 2009.

[9]. Ari Juels, "RFID Security and Privacy: A Research Survey", IEEE Journal on Selected Areas in Communications, Vol. 24, No. 2, 2006. 